\title{
Real Clinical Practice of Using Afatinib Therapy in NSCLC Patients with an Acquired EGFR T790M Mutation
}

\author{
KUNIHIKO MIYAZAKI ${ }^{1}$, TOMOHIRO TAMURA ${ }^{2}$, TAKAYUKI KABURAGI ${ }^{2}$, \\ KAZUHITO SAITO ${ }^{3}$, MASAHARU INAGAKI ${ }^{3}$, TAKAAKI YAMASHITA ${ }^{4}$, HIDEO ICHIMURA ${ }^{5}$, \\ TAKESHI NAWA ${ }^{5}$, TAKEO ENDO ${ }^{6}$, KENJI HAYASHIHARA ${ }^{7}$, MASAKI KIMURA $^{8}$, \\ KOICHI KURISHIMA ${ }^{9}$, HIROYUKI NAKAMURA ${ }^{10}$, KINYA FURUKAWA $^{10}$, \\ NORIHIRO KIKUCHI ${ }^{11}$, HIROAKI SATOH ${ }^{12}$ and NOBUYUKI HIZAWA ${ }^{13}$ \\ ${ }^{1}$ Division of Respiratory Medicine, Ryugasaki Saiseikai Hospital, Ryugasaki, Japan; \\ ${ }^{2}$ Respiratory Center, Ibaraki Prefectural Central Hospital, Kasama, Japan; \\ ${ }^{3}$ Divisions of Respiratory Medicine and Thoracic Surgery, Tsuchiura Kyodo General Hospital, Tsuchiura, Japan; \\ ${ }^{4}$ Division of Respiratory Medicine, JA Toride Medical Center, Toride, Japan; \\ ${ }^{5}$ Divisions of Respiratory Medicine and Thoracic Surgery, Hitachi General Hospital, Hitachi, Japan; \\ ${ }^{6}$ Division of Respiratory Medicine, Mito Medical Center, Mito, Japan; \\ ${ }^{7}$ Division of Respiratory Medicine, Ibarakihigashi Hospital, Tokai, Japan; \\ ${ }^{8}$ Division of Thoracic Surgery, Tsukuba Memorial Hospital, Tsukuba, Japan; \\ ${ }^{9}$ Division of Respiratory Medicine, Tsukuba Medical Center Hospital, Tsukuba, Japan; \\ ${ }^{10}$ Divisions of Respiratory Medicine and Thoracic Surgery, Tokyo Medical University, \\ Ibaraki Medical Center, Ami-machi, Japan; \\ ${ }^{11}$ Division of Respiratory Medicine, Kasumigaura Medical Center, Tsuchiura, Japan; \\ ${ }^{12}$ Division of Respiratory Medicine, Mito Kyodo General Hospital-Mito Medical Center, \\ University of Tsukuba, Mito, Japan; \\ ${ }^{13}$ Faculty of Clinical Medicine, University of Tsukuba, Tsukuba, Japan
}

\begin{abstract}
Background/Aim: To describe real clinical outcomes when using afatinib therapy to treat non-small cell lung cancer patients who have an acquired EGFR T790M mutation. Materials and Methods: A retrospective chart review was conducted from January 2013 to November 2017 sourced from 15 medical institutes that cover a population of three million people. Results: There were 74 patients who met the above-mentioned criteria. Treatment outcomes with afatinib, in patients with or without tyrosine kinase inhibitor (TKI) therapy prior to afatinib, were similar to previously reported clinical trials. Stratification of patients by the presence or absence of TKI pretreatment before afatinib, and the presence or absence of an acquired T790M mutation found no statistical difference in overall survival.
\end{abstract}

Correspondence to: Hiroaki Satoh, Division of Respiratory Medicine, Mito Kyodo General Hospital - Mito Medical Center, University of Tsukuba, Mito, Japan. E-mail: hirosato@md.tsukuba.ac.jp

Key Words: Clinical practice, afatinib, non-small cell lung cancer, acquired T790M mutation, osimertinib, immune-checkpoint inhibitor.
Conclusion: This population-based study found that the disadvantages of pretreatment before afatinib, and absence of an acquired T790M EGFR mutation, could be overcome by an appropriate treatment strategy in clinical practice.

The rapid development of effective mutation-specific treatments using epidermal growth factor receptor-tyrosine kinase inhibitors (EGFR-TKIs) has greatly advanced personalized medicine in non-small cell lung cancer (NSCLC) (1). Afatinib is a second-generation EGFR-TKI, proven to be superior to standard platinum-based chemotherapies as a first-line therapy in patients with EGFRmutant NSCLC (2). However, acquired resistance to EGFRTKIs such as afatinib led to the development of third generation EGFR-TKIs, such as osimertinib (3). A recent phase III clinical trial (FRAURA) (4) found improved efficacy of osimertinib over gefitinib and erlotinib as firstline treatment (4), but the trial did not directly compare afatinib with osimertinib as first-line treatment. As such, the optimal first-line therapy and exact sequence of EGFR-TKIs has yet to be fully determined. In recent years, real clinical practical outcomes have been reevaluated from the viewpoint of supplementing clinical trials. Although numerous clinical 
trials of afatinib therapy have or are being conducted (5-13), there are only a few reports of afatinib treatment in clinical practice (14-17). Furthermore, while providing important information, these clinical practice reports are limited by lack of survival data and EGFR T790M mutation status, short observation periods, and the inclusion of patients whose tumors do not express EGFR (14-17).

We previously performed a population-based multiinstitute study in 233 NSCLC patients with EGFR mutations. In this retrospective study, $34(45.3 \%)$ of 75 patients treated with afatinib had acquired an EGFR T790M resistance mutation (18). In the current study, we used this cohort of 74 patients to conduct a detailed investigation on the effects of afatinib treatment. We focused on issues raised from our experience in real clinical practice using this database.

\section{Patients and Methods}

Patients. This multi-institute, population-based study included patients with advanced EGFR-mutant NSCLC who had a T790M gene mutation test after the administration of one or more TKIs. We investigated effects and adverse events (AEs) among patients who received afatinib. Patients were sourced from 15 medical institutes in the Ibaraki prefecture between January 2013 and November 2017. The Ibaraki prefecture in Japan spans an area of $6,095 \mathrm{~km}^{2}$ and a population of three million people. Patients who had a T790M mutation at initial diagnosis were excluded from this study.

Ethics statement. This study was conducted in accordance with the Declaration of Helsinki and the Ethical Guidelines for Medical Research Involving Human Subjects in Japan. Informed consent for $E G F R$ mutational analysis was obtained from patients since this analysis was performed under the Japanese insurance system. This study was approved by the Institutional Review Board of Mito Kyodo General Hospital (no. 17-20) or independent ethics committees associated with each study institute.

T790M mutation analysis. Specimens were obtained from each patient who experienced relapse after the administration of one or more EGFR-TKIs. T790M mutation analysis was performed in biopsy specimens, cytology specimens, and plasma specimens using the assay method normally used in each study institute, such as the Cobas EGFR Mutation Test and allele-specific real-time polymerase chain reaction (PCR).

Treatment and assessments. Afatinib was prescribed as a continuous treatment at a starting dose of $40 \mathrm{mg} /$ day. Dose reductions were allowed based on patient tolerability. Treatment was continued in the absence of disease progression and/or unacceptable adverse effects (Response Evaluation Criteria in Solid Tumor (RECIST) version 1.1).

Statistical analysis. Differences in proportions between two independent groups were compared using the chi-square test. Patient survival time was calculated from the initiation of targeted therapy to death or last follow up. The survival rate was analyzed using the Kaplan-Meier method and comparisons were performed using the $\log$ rank test. A value of $p<0.05$ was considered statistically significant.
Table I. Characteristics of 74 patients with EGFR mutated-non-small cell lung cancer.

\begin{tabular}{lc}
\hline Age, years & Median: 66, range=35-85 \\
Gender & $32(43.2 \%) / 42(56.8 \%)$ \\
Male/Female & $29(39.2 \%) / 45(60.8 \%)$ \\
Smoking history & \\
Present/Absent & $27(36.5 \%) / 39(52.7 \%) /$ \\
Performance status & $8(10.8 \%)$ \\
0/1/2 or more & \\
& $73(98.6 \%)$ \\
Histology & $1(1.4 \%)$ \\
Adenocarcinoma & \\
Adenosquamous cell carcinoma & $10(13.5 \%)$ \\
Pathological stage & $64(86.5 \%)$ \\
III A-B & \\
IVA-B & $51(68.9 \%)$ \\
EGFR mutation & $18(24.3 \%)$ \\
Exon 19 deletion & $5(6.8 \%)$ \\
Exon 21 L858R & \\
Others & $48(64.9 \%)$ \\
Specimen types obtained & $26(35.1 \%)$ \\
Biopsy, cytology specimens & \\
Plasma specimens & $35(47.3 \%)$ \\
Acquired $E G F R$ T790M mutation & $39(52.7 \%)$ \\
Positive & \\
Negative & $33(44.6 \%)$ \\
Patients with prior afatinib treatment & $29(39.2 \%)$ \\
TKI naïve patients & $4(5.4 \%)$ \\
Received no therapy & $41(55.4 \%)$ \\
Received cytotoxic chemotherapy only & $20(27.0 \%)$ \\
Previously TKI-treated patients & $12(16.2 \%)$ \\
Received gefitinib & $9(12.2 \%)$ \\
Received erlotinib & \\
Received both first generation TKIs & \\
\hline & \\
\hline & \\
& \\
&
\end{tabular}

EGFR: Epidermal growth factor; TKI: tyrosine kinase inhibitor.

\section{Results}

Patient characteristics. This study comprised 233 patients from 15 study institutes. All patients had NSCLC with an EGFR mutation, and had undergone a T790M mutation test after acquiring resistance to EGFR-TKI. Among them, 74 patients from 13 study institutes were treated with afatinib and found to have acquired the T790M mutation. These 74 patients were included in this study and their characteristics are shown in Table I. There were 33 TKI-naïve patients (29 patients with no previous therapy and four patients with cytotoxic chemotherapy regimen only) and 41 patients previously treated with a first-generation TKI.

Effect of prior treatment with an EGFR TKI or chemotherapy on response to afatinib. We firstly compared the clinical outcomes of afatinib-treated patients who either did or did not receive prior treatment with an EGFR TKI. In the 33 TKI naïve patients ( 29 patients with no previous 
therapy and four patients with chemotherapy only), the overall response rate (ORR), disease control rate (DCR), and progression-free survival (PFS) were $69.7 \%, 84.8 \%$, and 13.0 months [95\% confidence interval $(95 \% \mathrm{CI})=9.3-$ 16.7 months], respectively. In the 41 patients previously treated with a first generation TKI; the ORR, DCR, and PFS were $36.6 \%, 80.5 \%$, and 5.0 months $(95 \% \mathrm{CI}=3.2-6.8$ months), respectively. The increase in PFS observed patients without previous TKI therapy compared with those with previous TKI therapy was statistically significant $(p=0.0138)$.

We next compared clinical outcomes in relation to the specific pretreatments; gefitinib, erlotinib, and chemotherapy. The ORR, DCR, and median PFS of the 20 patients whose previous treatment was gefitinib (G group) were $45 \%, 80 \%$, 5.0 (3.3-6.7) months, respectively. In the 12 patients whose previous treatment was erlotinib (E group), the ORR, DCR, and median PFS were 33\%, 75\%, 10.0 (1.0-23.6) months, respectively. In the nine patients whose previous treatment included both of these TKIs (both TKI group), the ORR, DCR, and median PFS were 22\%, 88.9\%, 5.0 (2.2-7.8) months, respectively. There was no difference in PFS among pretreatment groups: $\mathrm{G}$ group vs. E group: $p=0.4735, \mathrm{G}$ group $v s$. both TKI group: $p=0.6495$, and $\mathrm{E}$ group $v s$. both TKI group: $p=0.1817$.

Effect of the pre-treatment regimen on afatinib efficacy. In the 14 patients pretreated with chemotherapy, ORR, DCR, and PFS were $35.7 \%, 71.4 \%$, and 7.0 (range=2.2-11.8) months, respectively. In the 27 patients pretreated with a TKI, ORR and DCR, PFS were $37.0 \%, 85.2 \%$, and 5.0 (range=3.0-7.0) months, respectively. There was no significant difference in PFS between these two groups $(p=0.7107)$.

Detailed summary of treatments administered after afatinib therapy. Figure 1 outlines the various treatments administered to our cohort following afatinib treatment, with patients stratified by various genetic characteristics, response descriptors, and treatment regimens. Specifically, the 74 patients were stratified by the presence or absence of an acquired T790M mutation (Figure 1A), their EGFR mutation type (Figure 1B), their response to afatinib (Figure 1C), the median PFS of afatinib therapy (seven months) (Figure 1D), and the number of treatment regimens prior to afatinib (Figure 1E). Figure 1F shows the treatments in 51 patients who received treatment prior to afatinib therapy stratified by the median PFS prior to afatinib therapy (19 months).

Effect of osimertinib after afatinib. In 33 TKI naïve patients, 14 had an acquired EGFR T790M mutation and of these, 12 patients received osimertinib. The ORR, DCR, and median PFS were $75.0 \%, 100 \%$, and 10.0 (95\% CI=5.4-14.6) months, respectively. In 41 patients previously treated with a first line TKI, 21 had an acquired T790M mutation and of these, 19 received osimertinib. The ORR, DCR, and median PFS were $63.2 \%, 89.5 \%$, and $14.0 \quad(95 \% \mathrm{CI}=6.7-21.3)$ months, respectively. There was no significant difference in PFS between these two groups of patients $(p=0.7387)$.

Effect of an immune-checkpoint inhibitor after afatinib. Eleven patients (five TKI naïve patients and six patients previously treated with first line TKI) received immunecheckpoint inhibitor (ICPI) therapy after afatinib therapy. The ORR, DCR, and median PFS were $18.2 \%, 45.5 \%$, and $3.0(95 \% \mathrm{CI}=1.6-4.4)$ months, respectively. Two patients had PFS for more than seven months, the median PFS with afatinib therapy, and are still under treatment.

Treatment durations before and after afatinib. Sixteen of the 33 TKI naïve patients and 21 of the 41 patients previously treated with first line TKI died from the disease during the study period. Overall survival (OS) after the initiation of afatinib in these groups of patients was 26 (95\% CI=22.129.9) months and $30 \quad(95 \% \mathrm{CI}=21.9-34.1)$ months, respectively. There was no significant difference in OS between groups $(p=0.8341)$.

Figure 2 shows treatment durations before and after afatinib therapy. Patients were divided into four groups based on the presence or absence of EGFR TKI pretreatment before afatinib, and presence or absence of an acquired T790M mutation: 21 patients with T790M mutation who had TKI pretreatment before afatinib therapy (A), 20 patients without T790M mutation who had TKI pretreatment before afatinib therapy (B), 12 patients with T790M mutation who had no TKI pretreatment before afatinib therapy and 2 patients with T790M mutation who had chemotherapy only before afatinib therapy (C), and 17 patients without T790M mutation who had no TKI pretreatment before afatinib therapy and 2 patients without T790M mutation who had chemotherapy only before afatinib therapy (D). There was no statistically significant difference in OS among these four groups of patients. A vs. B: $p=0.4855$, A vs. C: $p=0.6742$, A vs. D: $p=0.3391, \mathrm{~B}$ vs. C: $p=0.9344$, B vs. D: $p=0.8049, \mathrm{C}$ vs. D: $p=0.8205$.

Adverse events. Nine patients discontinued afatinib treatment due to adverse events (AE) (lung toxicity in three patients, dermal toxicity in three patients, paronychia in one patient, abdominal pain in one patient, general fatigue in one patient, and faintness in one patient). All AEs were grade three or less (NCI toxicity criteria) and disappeared after stopping afatinib therapy. No patients deceased. All of them had additional treatment after afatinib and the median PFS of the treatments was 4 (95\% CI=1.1-6.9) months. 


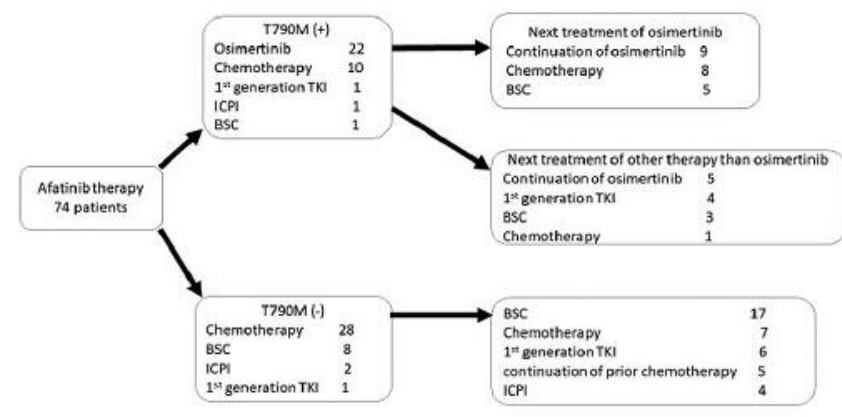

A

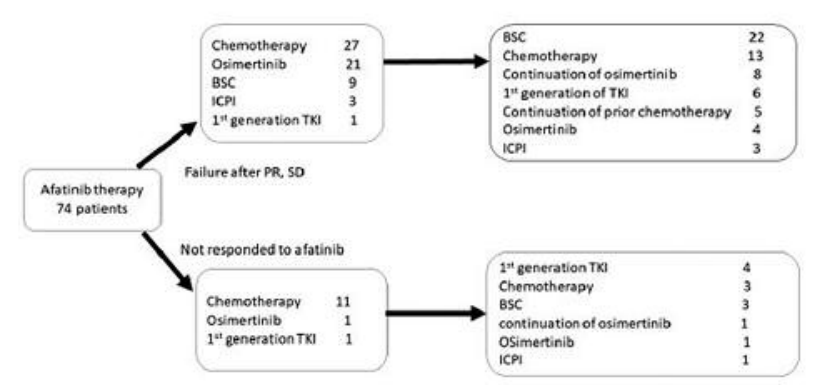

C

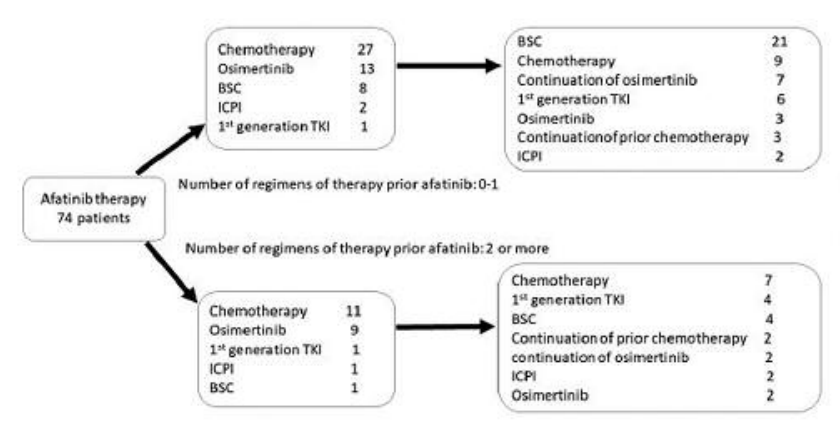

E

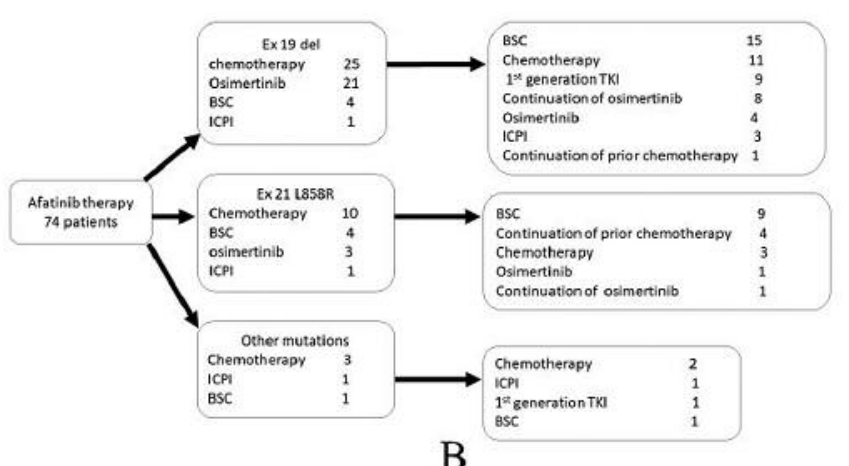

B

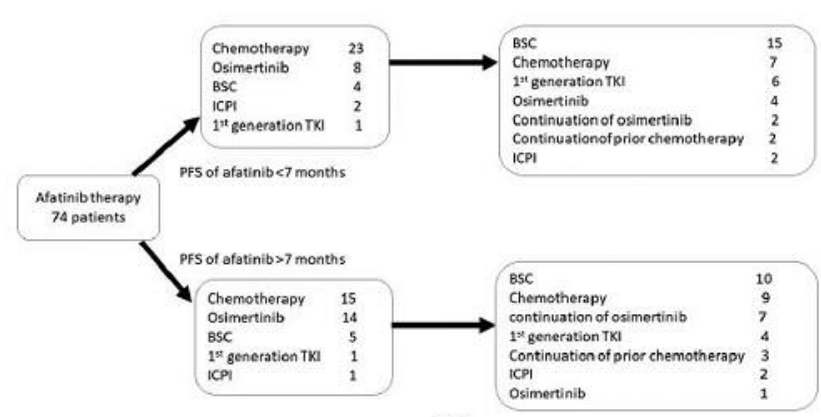

D

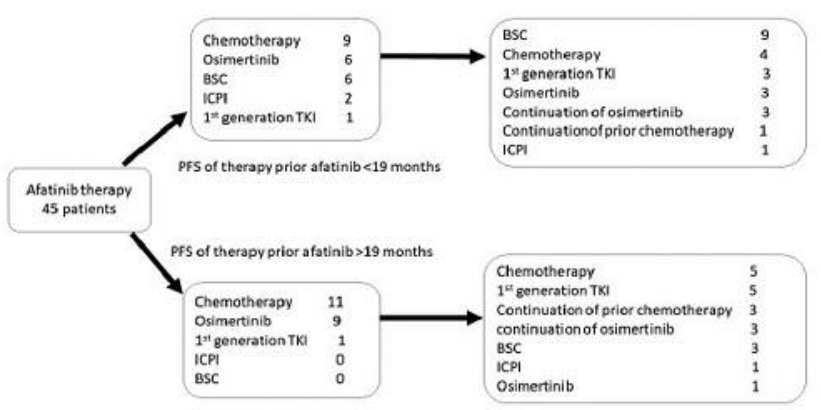

F

Figure 1. Details of treatment administered after afatinib treatment in our patient cohort. A: Treatments administered to 74 patients with or without an acquired EGFR T790M resistance mutation. B: Treatments administered to 74 patients according to their EGFR gene mutation type. C: Treatments administered to 74 patients stratified by their response to afatinib treatment. D: Treatments administered to 74 patients stratified by the median PFS of afatinib therapy (seven months). E: Treatments administered to 74 patients stratified by the number treatment regimens prior to afatinib. F: Treatments administered to 45 patients who received treatment prior to afatinib stratified by the median PFS prior to afatinib therapy (19 months).

\section{Discussion}

Similar to other antitumor agents, clinical trials using afatinib therapy have been conducted separately for treatment-naïve patients and those who have previously received other treatments, including EGFR-TKI (5-13). In these clinical trials, treatment-naïve patients had more favorable outcomes with afatinib therapy than those with previous treatment $[\mathrm{ORR}=62-70 \%, \mathrm{DCR}=83-91 \%, \mathrm{PFS}=11-$
12 months, and median OS 22-28 months in treatment naïve patients (5-9); ORR $=6-27 \%, \mathrm{DCR}=37-70 \%$, median $\mathrm{PFS}=2$ 8 months, and median OS 8-23 months in those with prior treatment (10-13)]. In the present study, the ORR, DCR, median PFS, and median OS following afatinib therapy in TKI-naïve patients were 70\%, 85\%, 13.0 (range=9.3-16.7) months, $26.0(95 \% \mathrm{CI}=22.1-29.9)$ months, respectively. In those who had previous TKI therapy, these indicators were $37 \%, \quad 81 \%, \quad 5.0(95 \% \mathrm{CI}=3.2-6.8)$ months, and 30.0 


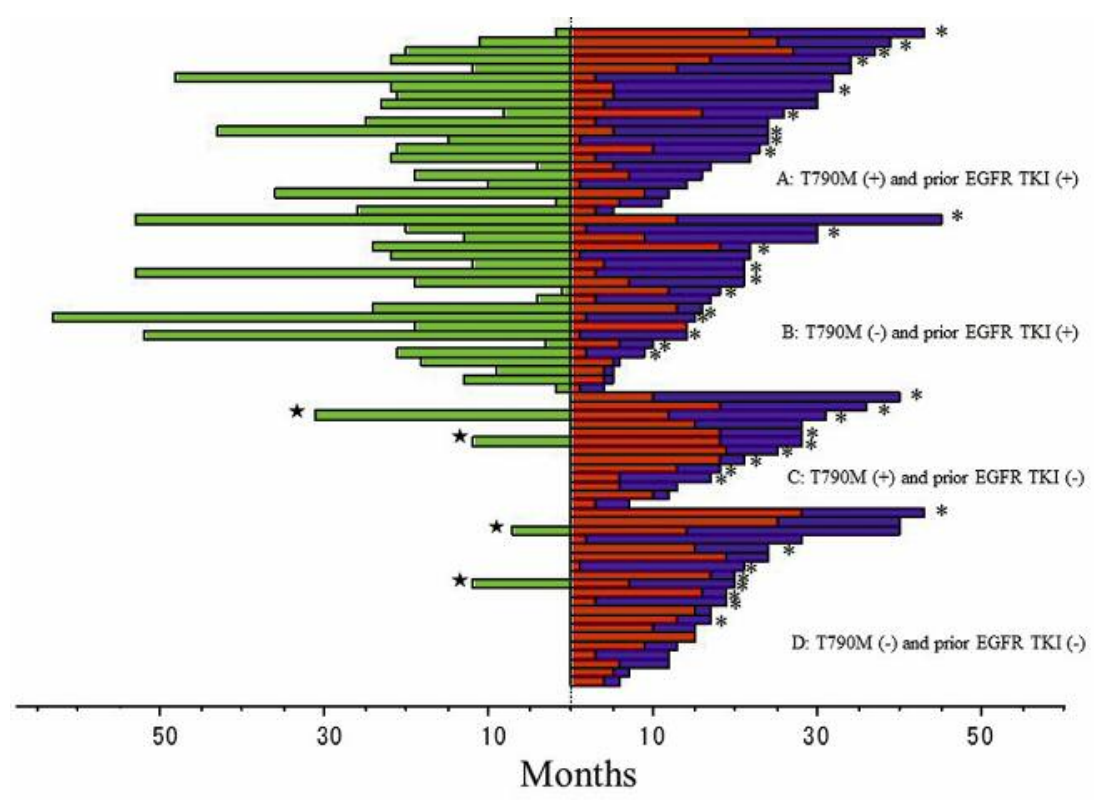

Figure 2. Treatment durations before and after afatinib therapy. A: 21 patients with T790M mutation who had TKI pretreatment before afatinib therapy. B: 20 patients without T790M mutation who had TKI pretreatment before afatinib therapy. C: 12 patients with T790M mutation who had no TKI pretreatment before afatinib therapy and 2 patients with T790M mutation who had chemotherapy only before afatinib therapy. D: 17 patients without T790M mutation who had no TKI pretreatment before afatinib therapy and 2 patients without T790M mutation who had chemotherapy only before afatinib therapy. Green bar: Treatment duration before afatinib therapy in each patient, red bar: PFS of afatinib therapy in each patient, blue bar: survival period after afatinib therapy in each patient. Asterisk: Censored patient; star: patient who had chemotherapy only before afatinib therapy. There was no statistically significant difference in OS among these four groups of patients. A vs. B: $p=0.4855, A v s . C: p=0.6742, A$ vs. D: $p=0.3391, B$ vs. $C: p=0.9344$, B vs. $D: p=0.8049, C$ vs. $D: p=0.8205$.

(95\% CI=21.9-34.1) months, respectively. Therefore, while our definition of pretreatment is somewhat different to earlier clinical trials, our results confirm that afatinib therapy in real clinical practice can achieve comparable results to that observed in clinical trials (5-13).

When comparing treatment outcomes between the current study and previous studies of real clinical practice, two previous reports describe an ORR of $26-27 \%$, a DCR of $67-$ $80 \%$, and a time to treatment failure (TTF) of 3.3-3.7 months $(15,17)$. Collectively, the results from real clinical practice (including ours) show a much higher response rate compared with an investigator assessment of 6.0-8.2\% in clinical trials $(10,11)$. Taken together, these results indicate afatinib might have moderate antitumor activity in some advanced NSCLC patients with acquired resistance to first-generation TKIs, although PFS and OS with afatinib therapy did not necessarily have satisfactory results.

Choi et al. recently reported that immediate pretreatment with pemetrexed monotherapy or a platinum doublet before afatinib therapy was associated with a longer TTF for afatinib (17). In our present study however, the type of treatment just prior to afatinib therapy, whether chemotherapy or TKI, did not affect PFS with afatinib. In addition, we found the type of TKI administered before afatinib, whether gefitinib, erlotinib, or both, and the type of EGFR gene mutation in the patient, whether EX19 mutation or EX21 mutation, did not affect PFS of afatinib. The inconsistencies between our study and that reported by Choi et al. are likely due to both studies analyzing only a small number of patients. Yet their observation of differences in response depending on the sequence of administration of drugs is noteworthy and highlights the need to use detailed medical history records.

Next, the effects of treatment conducted after afatinib therapy was evaluated. Some large scale clinical trials have reported median PFS of 10-13 months $(19,20)$ with osimertinib treatment after afatinib. We showed that the median PFS with osimertinib treatment after afatinib was comparable (10 months; 95\% $\mathrm{CI}=5.4-14.6)$. In regards to adjuvant ICPI, the clinical indicators in our patients treated with ICPI after afatinib were not promising $(\mathrm{ORR}=18 \%$, $\mathrm{DCR}=46 \%$, and median PFS $3.0 \quad(95 \% \mathrm{CI}=1.6-4.4)$ months). However, there was a subset of patients in this group with PFS of more than 7 months, the median PFS with afatinib therapy. When patients were stratified by TKI pretreatment before afatinib and an acquired T790M gene 
mutation, there was no significant difference in OS among these four groups. Unexpectedly, in this study, the presence or absence of first generation TKI pretreatment, and the presence or absence of an acquired T790M gene expression did not affect OS. Thus far, there have been no studies showing no difference in OS in a real clinical practice situation. The reason for this outcome is not clear. It could be the result of the small number of patients and/or the short follow up period. Either way, the importance of selecting "appropriate treatment" properly and implementing it adequately even in clinically disadvantageous situations was reconfirmed. This suggests the possibility of overcoming disadvantages associated with pretreatment before afatinib therapy, absence of an acquired T790M gene mutation, and/or osimertinib therapy with appropriate treatment strategies.

In this study nine patients discontinued afatinib treatment due to AEs. Lung toxicity was observed in three patients, and it developed at two, three, and six months from the initiation of administration. Abdominal pain, general fatigue, and faintness occurred in all patients within two months after the initiation of afatinib therapy. Dermal toxicity leading to discontinuation of afatinib developed six months after the start of treatment in all but one patient. AEs in all patients were grade three or lower, and there were no deceased patients. All of them had additional treatment after afatinib with a median PFS of four months.

This population-based, multi-institute study covering a single prefecture has several limitations. It was a retrospective study with a small number of patients from varied backgrounds. Although all the patients were positive for $E G F R$ mutations and were evaluated for the acquired EGFR T790M mutation after afatinib administration, this study included patients with different treatment histories. In particular, the treatments before and after afatinib treatment varied. However, this study has clinical significance as it reflects real practice without selection bias. As such, our results should be used to complement clinical trial results when patients are collected with a selection bias.

In conclusion, the efficacy and AEs of afatinib therapy in EGFR-TKI-naïve patients and those with previous TKI therapy was evaluated. In addition, the effects of pretreatment and post-treatment on afatinib therapy were investigated. Treatment durations including before and after afatinib therapy were also shown. Based on the results of large-scale clinical trials that showed the superiority of osimertinib over first generation TKIs, an era will come when osimertinib can be administered as first line treatment. Even then, it is important to understand not only the results on patients undergoing clinical trials, but also the results in real clinical practice in order to carry out optimal treatment strategies for EGFR mutant patients.

\section{Conflicts of Interest}

No Authors have any conflict of interest to disclose regarding this study.

\section{References}

1 Villaruz LC, Burns TF, Ramfidis VS and Socinski MA: Personalizing therapy in advanced non-small cell lung cancer. Semin Respir Crit Care Med 34: 822-836, 2013.

2 Marquez-Medina D and Popat S: Afatinib: a second-generation EGF receptor and ErbB tyrosine kinase inhibitor for the treatment of advanced non-small-cell lung cancer. Future Oncol 11: 2525-2540, 2015.

3 Lamb YN and Scott LJ: Osimertinib: A Review in T790Mpositive advanced non-small cell lung cancer. Target Oncol 12: 555-562, 2017.

4 Soria JC, Ohe Y, Vansteenkiste J, Reungwetwattana T, Chewaskulyong B, Lee KH, Dechaphunkul A, Imamura F, Nogami N, Kurata T, Okamoto I, Zhou C, Cho BC, Cheng Y, Cho EK, Voon PJ, Planchard D, Su WC, Gray JE, Lee SM, Hodge R, Marotti M, Rukazenkov Y and Ramalingam SS; FLAURA Investigators: Osimertinib in untreated EGFR-mutated advanced non-small-cell lung cancer. N Engl J Med 378: 113-125, 2018.

5 Yang JC, Shih JY, Su WC, Hsia TC, Tsai CM, Ou SH, Yu CJ, Chang GC, Ho CL, Sequist LV, Dudek AZ, Shahidi M, Cong XJ, Lorence RM, Yang PC and Miller VA: Afatinib for patients with lung adenocarcinoma and epidermal growth factor receptor mutations (LUX-Lung 2): A phase 2 trial. Lancet Oncol 13: 539548, 2018.

6 Sequist LV, Yang JC, Yamamoto N, O'Byrne K, Hirsh V, Mok T, Geater SL, Orlov S, Tsai CM, Boyer M, Su WC, Bennouna J, Kato T, Gorbunova V, Lee KH, Shah R, Massey D, Zazulina V, Shahidi M and Schuler M: J Clin Oncol 31: 3327-3334, 2013.

7 Wu YL, Zhou C, Hu CP, Feng J, Lu S, Huang Y, Li W, Hou M, Shi JH, Lee KY, Xu CR, Massey D, Kim M, Shi Y and Geater SL: Afatinib versus cisplatin plus gemcitabine for first-line treatment of Asian patients with advanced non-small-cell lung cancer harbouring EGFR mutations (LUX-Lung 6): an openlabel, randomised phase 3 trial. Lancet Oncol 15: 213-222, 2014.

8 Park K, Tan EH, O'Byrne K, Zhang L, Boyer M, Mok T, Hirsh V, Yang JC, Lee KH, Lu S, Shi Y, Kim SW, Laskin J, Kim DW, Arvis CD, Kölbeck K, Laurie SA, Tsai CM, Shahidi M, Kim M, Massey D, Zazulina V and Paz-Ares L: Afatinib versus gefitinib as first-line treatment of patients with EGFR mutation-positive non-small-cell lung cancer (LUX-Lung 7): a phase 2B, open-label, randomised controlled trial. Lancet Oncol 17: 577-589, 2016.

9 Paz-Ares L, Tan EH, O'Byrne K, Zhang L, Hirsh V, Boyer M, Yang JC, Mok T, Lee KH, Lu S, Shi Y, Lee DH, Laskin J, Kim DW, Laurie SA, Kölbeck K, Fan J, Dodd N, Märten A and Park $\mathrm{K}$ : Afatinib versus gefitinib in patients with EGFR mutationpositive advanced non-small-cell lung cancer: overall survival data from the phase IIb LUX-Lung 7 trial. Ann Oncol 28: 270277, 2017.

10 Katakami N, Atagi S, Goto K, Hida T, Horai T, Inoue A, Ichinose Y, Koboyashi K, Takeda K, Kiura K, Nishio K, Seki Y, Ebisawa R, Shahidi M and Yamamoto N: LUX-Lung 4: a phase II trial of afatinib in patients with advanced non-small-cell lung cancer who progressed during prior treatment with erlotinib, gefitinib, or both. J Clin Oncol 31: 3335-3341, 2013. 
11 Soria JC, Felip E, Cobo M, Lu S, Syrigos K, Lee KH, Göker E, Georgoulias V, Li W, Isla D, Guclu SZ, Morabito A, Min YJ, Ardizzoni A, Gadgeel SM, Wang B, Chand VK and Goss GD; LUX-Lung 8 Investigators: Afatinib versus erlotinib as secondline treatment of patients with advanced squamous cell carcinoma of the lung (LUX-Lung 8): an open-label randomised controlled phase 3 trial. Lancet Oncol 16: 897-907, 2015.

12 Choi MK, Ahn JS, Kim YC, Cho BC, Oh IJ, Kim SW, Lee JS, Kim JH, Ahn MJ and Park K: Afatinib in heavily pretreated advanced NSCLC patients who progressed following prior gefitinib or erlotinib: Compassionate use program in Korea. Lung Cancer 119: 36-41, 2018.

13 Yang JC, Shih JY, Su WC, Hsia TC, Tsai CM, Ou SH, Yu CJ, Chang GC, Ho CL, Sequist LV, Dudek AZ, Shahidi M, Cong XJ, Lorence RM, Yang PC and Miller VA: Afatinib for patients with lung adenocarcinoma and epidermal growth factor receptor mutations (LUX-Lung 2): a phase 2 trial. Lancet Oncol 13: 539$548,2012$.

14 Wada Y, Koyama S, Kuraishi H, Miyahara T, Yoshiike F, Agatsuma T, Yamamoto R, Ono Y, Suzuki T, Hachiya T, Gomi D, Tateishi K, Hanaoka M and Koizumi T: Clinical analysis of patients treated with afatinib for advanced non-small cell lung cancer: A Nagano Lung Cancer Research Group observational study. Respir Investig 54: 462-467, 2016.

15 Schuler M, Fischer JR, Grohé C, Gütz S, Thomas M, Kimmich M, Schneider CP, Laack E and Märten A; Afatinib Compassionate Use Consortium: Experience with afatinib in patients with non-small cell lung cancer progressing after clinical benefit from gefitinib and erlotinib. Oncologist 19: 11001109,2014

16 Liang SK, Hsieh MS, Lee MR, Keng LT, Ko JC and Shih JY: Real-world experience of afatinib as a first-line therapy for advanced EGFR mutation-positive lung adenocarcinoma. Oncotarget 8: 90430-90443, 2017.
17 Choi MK, Ahn JS, Kim YC, Cho BC, Oh IJ, Kim SW, Lee JS, Kim JH, Ahn MJ and Park K: Afatinib in heavily pretreated advanced NSCLC patients who progressed following prior gefitinib or erlotinib: Compassionate use program in Korea. Lung Cancer 119: 36-41, 2018.

18 Kaburagi T, Kiyoshima M, Nawa T, Ichimura H, Saito T, Hayashihara K, Yamada H, Satoh H, Endo T, Inage Y, Saito K, Inagaki M, Hizawa N, Sato Y, Ishikawa H, Sakai M, Kamiyama K, Kikuchi N, Nakamura H, Furukawa K, Kodama T, Yamashita T, Nomura A and Yoshida S: Acquired EGFR T790M mutation after relapse following EGFR-TKI therapy: A population-based multi-institutional study. Anticancer Res 38: 3145-3150, 2018.

19 Mok TS, Wu YL, Ahn MJ, Garassino MC, Kim HR, Ramalingam SS, Shepherd FA, He Y, Akamatsu H, Theelen WS, Lee CK, Sebastian M, Templeton A, Mann H, Marotti M, Ghiorghiu $S$ and Papadimitrakopoulou VA; AURA3 Investigators: Osimertinib or platinum-pemetrexed in EGFR T790M-positive lung cancer. N Engl J Med 376: 629-640, 2017.

20 Akamatsu H, Katakami N, Okamoto I, Kato T, Kim YH, Imamura F, Shinkai M, Hodge RA, Uchida $\mathrm{H}$ and Hida T: Osimertinib in Japanese patients with EGFR T790M mutationpositive advanced non-small-cell lung cancer: AURA3 trial. Cancer Sci 109: 1930-1938, 2018.
Received July 14, 2018

Revised July 23, 2018

Accepted August 2, 2018 